\title{
Proton pump inhibitors and gastroenteritis
}

\author{
Robert-Jan Hassing ${ }^{1,2}$ - Annelies Verbon ${ }^{3} \cdot$ Herman de Visser $^{4} \cdot$ Albert Hofman $^{1}$. \\ Bruno H. Stricker ${ }^{1,5}$
}

Received: 2 November 2015/Accepted: 1 March 2016/Published online: 10 March 2016

(c) The Author(s) 2016. This article is published with open access at Springerlink.com

\begin{abstract}
An association between proton pump inhibitor (PPI) therapy and bacterial gastroenteritis has been suggested as well as contradicted. The aim of this study was to examine the association between the use of PPIs and occurrence of bacterial gastroenteritis in the prospective Rotterdam Study. The Rotterdam Study is a populationbased cohort study among 14,926 subjects aged 45 years and older with up to 24 years of follow-up. Analyses were performed with a generalized estimating equations method in participants who handed-in a diagnostic stool sample. Furthermore, a nested case-control analysis was performed using the total cohort as a reference group. A bacterial microorganism was isolated in 125 samples, whereas 1174 samples were culture negative. In the generalized estimating equations analysis, we found that participants with a bacterial gastroenteritis were more likely than controls to be current users of PPIs (adjusted OR 1.94; $95 \%$ CI 1.15-3.25). Different sensitivity analyses did not change this result. A considerably higher effect was observed (adjusted OR 6.14; $95 \%$ CI 3.81-9.91), using the total
\end{abstract}

Bruno H. Stricker

b.stricker@erasmusmc.nl

1 Department of Epidemiology, Erasmus Medical Centre, Wytemaweg 80, 3015 CN Rotterdam, The Netherlands

2 Department of Internal Medicine, Rijnstate Hospital, Arnhem, The Netherlands

3 Department of Medical Microbiology and Infectious Diseases, Erasmus Medical Centre, Rotterdam, The Netherlands

4 Star Medisch Diagnostisch Centrum, Rotterdam, The Netherlands

5 Inspectorate of Health Care, Utrecht, The Netherlands cohort as a reference in a nested case-control analysis. Current PPI therapy is associated with an increased risk of bacterial gastroenteritis. However, by reducing the risk of selection and information bias in our study design, we demonstrated that the effect is lower than previously assumed.

Keywords Gastroenteritis - Proton pump inhibitor . Campylobacter $\cdot$ Salmonella $\cdot$ Cohort study

\section{Introduction}

Proton pump inhibitors (PPIs) are among the most frequently prescribed drugs worldwide, but seem to be associated with an increased risk of bacterial gastroenteritis [19]. As a result warnings are introduced for people using PPIs, such as avoiding raw meat consumption and antibiotic treatment on demand for travels to the tropics, to prevent food borne infections. This cohort study was designed to asses if the magnitude of this risk, warrants preventive recommendations.

Common indications for PPIs are dyspepsia, peptic ulcer disease, reflux esophagitis, and Barrett's oesophagus [10]. PPIs reduce gastric acid production by up to $99 \%$ by irreversible blocking of $\mathrm{H}+/ \mathrm{K}+$ ATPase of parietal cells in the stomach [11]. They have a maximal effect within 4 days and the effect may persist up to 3 days after stopping use [12]. Associations between PPIs and infectious adverse events such as pneumonia, Clostridium difficileassociated diarrhoea and bacterial gastroenteritis have often been described [1-9, 13-17]. An increased risk of gastroenteritis might be explained by the strong reduction in gastric acid resulting in increased susceptibility to bacterial infections. Exogenous bacteria are usually destroyed 
in the stomach when the $\mathrm{pH}$ is $<3.0$. For species such as Vibrio cholerae and Campylobacter jejuni it has been shown in vitro that they are very sensitive to $\mathrm{pH}$ [18]. However, Salmonella species have been found to respond to low $\mathrm{pH}$ by developing adaptive mechanisms that allow survival in acid environments [19]. Furthermore, PPIs change the gut flora, which provides a homeostatic protection against ingested pathogens [20, 21]. PPIs also reduce the antibacterial activity of neutrophils which may facilitate Salmonella and Campylobacter infections [22, 23]. Several case-control studies have shown an increased risk of acquiring gastrointestinal infections caused by Campylobacter or Salmonella species in patients using PPIs [1-8]. In these case-control studies, a relatively high adjusted odds ratio (aOR) or relative risk was observed, ranging from 2.9 to 11.7 . In one nested case-control study, in which participants with a gastroenteritis prior to first PPI prescription were excluded, a considerably lower effect was observed (aOR 1.6) [9]. It has even been stated that there is no evidence that PPIs are associated with gastrointestinal infections based on outcomes adjusted for pretreatment susceptibility to bacterial gastrointestinal infections and time-dependent confounding factors [24], which observation suggests that previous case-control studies have suffered from selection or information bias. Therefore, we designed a nested case control study within The Rotterdam Cohort, a prospective cohort study, to examine the association between the use of PPIs and occurrence of bacterial gastroenteritis. To minimize the risk of information bias we used participants with negative stool samples as a control group. To test the hypothesis that an incorrect control group will influence the study results we also analysed the association using the total cohort as a control group.

\section{Materials and methods}

\section{Study population}

The study was performed in The Rotterdam Study, a prospective population-based cohort study in 14,926 people aged $\geq 45$ years, from one district (Ommoord) in the city of Rotterdam, the Netherlands [25]. In short, from 1990 through 1993, 7983 participants were included (cohort I). In 2000 an additional 3011 participants who had become 55 years old or older or who had moved into the district, were enrolled (cohort II). In 2006 another 3932 participants, aged 45 years and older were included (cohort III). Follow-up examinations are conducted every 4-5 years. Participants are continuously monitored through linkage of records from general practitioners.
The Rotterdam Study was approved by the medical ethics committee according to the Wet Bevolkingsonderzoek ERGO (Population Study Act Rotterdam Study) executed by the Ministry of Health, Welfare and Sports of the Netherlands. All study participants provided written informed consent.

\section{Definition of outcome}

A case was defined as a community-dwelling non-hospitalized individual with a positive stool sample for Campylobacter, Salmonella, Yersinia or Shigella species. A control was defined as an individual with a negative stool sample. Stool sample results were obtained from Star Medisch Diagnostisch Centrum (Star-MDC), a centre for medical diagnostics for outpatients in the city of Rotterdam. The majority of all laboratory tests, including microbiology tests, of patients from general practitioners within the Ommoord district of Rotterdam are performed at Star-MDC. Of all participants of The Rotterdam Study, of whom informed consent was obtained for requesting medical information, positive and negative microbiology tests between 1999 and April 2013 were obtained. Stool samples were selected and samples in which parasites were isolated were excluded. Detection of bacterial enteric pathogens in stool samples at Star-MDC is performed by Multiplex polymerase chain reaction (PCR), followed by culture and microscopy in case of a positive result. Until December 2010, when PCR was introduced at Star-MDC, detection of bacterial enteric pathogens was performed by conventional culture and microscopy only.

\section{Assessment of exposure and covariables}

Participants were considered as current user of PPI if the calendar date of the stool sample fell within a prescription episode of a PPI. Prescription episodes were calculated by dividing the total number of supplied pills by the recommended daily number. Additional covariables assessed were age, sex, cohort, calendar date (year), BMI, household status, past use of proton pump inhibitors, current or past use of H2-receptor antagonists, current use of chronic medication (antidiabetic medication, antihypertensive medication, or statins), intestinal anti-inflammatory agents, corticosteroids, immunosuppressant medication, meat consumption, red meat consumption, chicken consumption, egg consumption and alcohol consumption (for all dietary variables: yes/no and gram/days).

BMI and household status was obtained from baseline characteristics of The Rotterdam Study. Medication use was obtained through automated linkage with pharmacy filled prescription data, available from January 1st, 1991 until April 2013. Dietary data were available from 1 week 
food consumption questionnaires obtained at the first visit of cohort I and cohort III and at the third visit of cohort II. Multiple imputation $(10 \times)$ of missing dietary data and BMI was performed using all covariables.

\section{Statistical analyses}

\section{Model design}

To assess the association between current use of PPIs and gastrointestinal infections generalized estimating equations (GEE) was used to adjust for correlation between repeated measurements in the same participants, using a varying between-measurement time window [26, 27]. The working correlation matrix in which the model had the lowest quasi likelihood under independence model criterion was selected. The model was adjusted for age and sex and additionally for covariables changing the point estimate $(\beta)$ of current PPI use by more than $10 \%$ or if considered clinically relevant.

\section{Sensitivity analyses}

Different sensitivity analyses were performed. To exclude confounding by indication or protopathic bias we recoded PPI use started within 14 days before a positive stool sample as non-use. To take into account that in many occasions PPIs are not used on a daily basis, and therefore the actual period of exposure will probably exceed the period calculated based on the pharmacy data, we also included use during the past 14 and 30 days as potentially exposed cases in sensitivity analyses. To exclude confounding by contra-indication we censored every participant at the first case in a sensitivity analysis. Participants receiving medication from other sources than the pharmacy, such as nursing home residents, might introduce information bias. Therefore we did a sensitivity analysis excluding participants without pharmacy prescriptions during the last 90 days because nursing home residents do not obtain medication through a community pharmacy. Furthermore, we performed a sensitivity analysis for Campylobacter species and Campylobacter or Salmonella species only. Different analyses were stratified on sex.

\section{Additional analysis}

We also analysed the exposure of PPIs in a nested casecontrol analysis to assess the association between PPIs and gastrointestinal infections in the total population of The Rotterdam Study. The use of PPIs was used as a timevarying determinant of exposure as previously described [28]. To use the total population of The Rotterdam Study every case (participant with a positive stool sample) was matched to every other participant alive and eligible at the same calendar date (day). Each time a case was identified, exposure in this case was compared with exposure in the other participants (cases might become controls, and controls might become cases). The model was adjusted for age and sex and additionally for covariables used in the final model.

A two-sided $p$ value below 0.05 was considered statistically significant. Statistical analyses were performed using SPSS version 21.0 (IBM Corp., Somers, NY, USA).

\section{Results}

During the study period, 1329 stool cultures of participants of The Rotterdam Study were identified at Star-MDC. We excluded 30 stool samples because of isolation of a parasite, resulting in 1299 stool samples for the study. A bacterial microorganism was isolated in 125 samples, whereas 1174 samples were culture negative. In total $105(84.0 \%)$ Campylobacter species, 16 (12.8\%) Salmonella species, 3 (2.4\%) Yersinia species and $1(0.8 \%)$ Shigella sonnei were isolated. All 125 positive stool samples were collected from 118 different participants and all 1174 negative stool samples from 903 different participants. The percentage of missing data of BMI was $7.6 \%$ and of dietary data $28.6 \%$. Characteristics of cases and controls are shown in Table 1.

\section{Model design}

For the generalized estimating equations, the independent working correlation structure had the best fit. Designing the final model, past use of H2-receptor antagonists increased the point estimate $(\beta)$ of current PPI use, adjusted for age and sex, by more than $10 \%$, whereas current use of chronic medication, all decreased the effect by more than $10 \%$. We included age, sex, cohort, calendar date, past use of proton pump inhibitors, past use of H2-receptor antagonists, and current use of chronic medication in the final model (model 2). In the final model we found that participants with a bacterial gastroenteritis were more likely than control participants to be current users of PPIs, with an aOR of 1.94 (95\% CI 1.15-3.25) (Table 2).

\section{Sensitivity analyses}

Different sensitivity analyses did not result in a significant change of the effect (Table 2). A sensitivity analysis including use during the past 14 and 30 days resulted in aORs of 2.14 (95\% CI 1.35-3.38) and 2.28 (95\% CI 1.46-3.55), respectively. Excluding current use of PPIs for 14 days or more, to exclude confounding by indication or 
Table 1 Baseline characteristics of participants with stool sample

\begin{tabular}{|c|c|c|}
\hline & Participants with positive stool sample & Participants with negative stool sample \\
\hline Total & $118^{*}$ & 903 \\
\hline Age-year (SD) & $65.1 \pm 10.3$ & $68.1 \pm 12.8$ \\
\hline Male sex-no. (\%) & $49(41.5)$ & $301(33.3)$ \\
\hline \multicolumn{3}{|l|}{ Cohort (\%) } \\
\hline I & $34(28.8)$ & $325(36.0)$ \\
\hline II & $35(29.7)$ & $227(25.1)$ \\
\hline III & $49(41.5)$ & $351(38.9)$ \\
\hline Household alone-no. (\%) & $20(16.9)$ & $229(25.4)$ \\
\hline $\mathrm{BMI}^{*}(\mathrm{SD})$ & $25.2 \pm 4.2$ & $24.5 \pm 4.3$ \\
\hline Use of proton pump inhibitors-no. (\%) & $43(36.4)$ & $242(26.8)$ \\
\hline Current use for 14 days or more & $32(27.1)$ & $152(16.8)$ \\
\hline Including use during past 14 days & $50(42.4)$ & $269(29.8)$ \\
\hline Including use during past 30 days & $55(46.6)$ & $288(31.9)$ \\
\hline$>1$ Defined daily dose/day & $13(30.2)$ & $76(31.4)$ \\
\hline Past use only & $33(28.0)$ & $289(32.0)$ \\
\hline \multicolumn{3}{|l|}{ Medication use-no. (\%) } \\
\hline $\mathrm{H} 2$-receptor antagonists & $3(2.5)$ & $22(2.4)$ \\
\hline H2-receptor antagonists-past use & $37(31.4)$ & $312(34.6)$ \\
\hline Antidiabetic medication & $12(10.2)$ & $93(10.3)$ \\
\hline Antihypertensive medication & $50(42.4)$ & $316(35.0)$ \\
\hline Statins & $27(22.9)$ & $151(16.7)$ \\
\hline Antidiabetic, antihypertensive medication or statins & $55(46.6)$ & $376(41.6)$ \\
\hline Intestinal anti-inflammatory agents & $1(0.8)$ & $4(0.4)$ \\
\hline Corticosteroids & $3(2.5)$ & $15(1.7)$ \\
\hline Immunosuppressant medication & 0 & $6(0.7)$ \\
\hline \multicolumn{3}{|l|}{ Dietary—no. (\%) } \\
\hline No meat consumer ${ }^{\dagger}$ & $2(2.4)$ & $6(0.7)$ \\
\hline No red meat consumer $\Pi$ & $2(2.4)$ & $11(1.2)$ \\
\hline No chicken consumer $\Pi$ & $15(18.3)$ & $105(11.6)$ \\
\hline No egg consumer $\Pi$ & $5(6.1)$ & $48(5.3)$ \\
\hline Alcohol $^{\$}$ & $72(61.0)$ & $558(61.8)$ \\
\hline \multicolumn{3}{|l|}{ Dietary—gram/days $(S D)$} \\
\hline Meat $^{\dagger}$ & $110.9 \pm 60.4$ & $103.3 \pm 54.4$ \\
\hline Red meat $\Pi$ & $91.7 \pm 55.0$ & $85.5 \pm 50.0$ \\
\hline Chicken $^{\Pi}$ & $17.1 \pm 18.1$ & $16.4 \pm 16.5$ \\
\hline $\operatorname{Eggs}^{\Pi}$ & $15.0 \pm 11.1$ & $15.7 \pm 12.9$ \\
\hline Alcohol $^{\$}$ & $13.0 \pm 15.7$ & $13.4 \pm 15.2$ \\
\hline
\end{tabular}

* Out of total 125 positive isolates

${ }^{\dagger}$ Patients with positive stool sample $N=82$, negative stool sample $N=644$

* Patients with positive stool sample $N=111$, negative stool sample $N=839$

$\Pi$ Patients with positive stool sample $N=82$, negative stool sample $N=646$

$\$$ Patients with positive stool sample $N=93$, negative stool sample $N=712$

protopathic bias, resulted in an aOR of 1.99 (95\% CI 1.19-3.35). Censoring at the first case, to exclude confounding by contra-indication, resulted in an aOR of 2.02 (95\% CI 1.19-3.42) and excluding participants without prescriptions during the last 90 days in an aOR of 1.78 (95\% CI 1.05-3.01). An aOR of 1.93 (95\% CI 1.11-3.36) and 2.05 (95\% CI 1.20-3.49) was observed in sensitivity analyses for including only Campylobacter and 
Table 2 Association of use of proton pump inhibitors with bacterial gastrointestinal infections

\begin{tabular}{|c|c|c|c|c|c|}
\hline & Number of cases & Number in cohort & Use of PPI (\%) & $\begin{array}{l}\text { Cases } N=125 \\
\text { OR }(95 \% \mathrm{CI})\end{array}$ & $P$ value \\
\hline \multicolumn{6}{|l|}{ Current use of proton pump inhibitor } \\
\hline Univariate analysis & 125 & 1299 & $375(28.9)$ & $1.50(1.01 ; 2.23)$ & 0.047 \\
\hline Adjusted analysis—model 1 & 125 & 1299 & $375(28.9)$ & $1.62(1.09 ; 2.43)$ & 0.018 \\
\hline Adjusted analysis-model 2 & 125 & 1299 & $375(28.9)$ & $1.94(1.15 ; 3.25)$ & 0.013 \\
\hline \multicolumn{6}{|l|}{ Sensitivity analyses-model 2} \\
\hline Only including current use for 14 days or more & 125 & 1299 & $242(18.2)$ & $1.99(1.19 ; 3.35)$ & 0.009 \\
\hline Including use during past 14 days & 125 & 1299 & $422(32.5)$ & $2.14(1.35 ; 3.38)$ & 0.001 \\
\hline Including use during past 30 days & 125 & 1299 & $453(34.9)$ & $2.28(1.46 ; 3.55)$ & $<0.001$ \\
\hline Censored at first case & 118 & 1246 & $353(28.3)$ & $2.02(1.19 ; 3.42)$ & 0.009 \\
\hline Excluding no medication for $>90$ days & 124 & 1223 & $375(30.7)$ & $1.78(1.05 ; 3.01)$ & 0.032 \\
\hline Campylobacter only & 105 & 1279 & $368(28.8)$ & $1.93(1.11 ; 3.36)$ & 0.019 \\
\hline Campylobacter and Salmonella & 121 & 1295 & $375(29.0)$ & $2.05(1.20 ; 3.49)$ & 0.008 \\
\hline Male only & 53 & 436 & $131(30.0)$ & $3.28(1.44 ; 7.49)$ & 0.005 \\
\hline Female only & 72 & 863 & $244(28.3)$ & $1.31(0.66 ; 2.60)$ & 0.45 \\
\hline
\end{tabular}

Generalized estimating equations method, negative stool cultures as control group

Model 1: adjusted for sex, age

Model 2: adjusted for sex, age, cohort, calendar date, past use of proton pump inhibitors, current use of chronic medication, past use of H2receptor antagonists

Campylobacter or Salmonella, respectively (Table 2). After stratifying on sex a difference was observed between male (aOR 3.28; $95 \%$ CI 1.44-7.49) and female (aOR $1.31 ; 95 \%$ CI $0.66 ; 2.60)$ participants (Table 2).

\section{Additional analysis}

In a matched case-control analysis, adjusting for the same covariables of the final model, but using all other participants of The Rotterdam Study as control group, a considerably higher aOR of 6.14 (95\% CI 3.81-9.91) was observed compared to the GEE analysis using negative stool samples as control group (Table 3).

\section{Discussion}

PPIs have been associated with an increased risk of bacterial gastroenteritis in previous studies [1-9]. In this population based cohort study we found that current PPI therapy was associated with a strongly increased risk of bacterial gastroenteritis, with an aOR of more than six. However, this risk decreased to 1.94 after restriction to the subgroup with stool samples. Therefore, we suspect that information bias inflated the risks in other population based studies which have shown an association between PPI therapy and an increased risk of Campylobacter and Salmonella infections before. Although, people using PPIs still should be careful consuming food which could be contaminated, such as beef, poultry or processed food, the risk of gastroenteritis is probably less than previously assumed.

One of the strengths of our study is that we tried to use a comparable control group by only using participants with negative stool samples. In most of the previous population based studies the magnitude of the effect may have been overestimated as a result of the use of incomparable control groups. Typically, case control studies regarding use of PPIs may suffer from a "healthy control" bias. We further tried to avoid healthy control bias by correcting for the use of chronic medication. Some of the previous studies used a random sample of population registries, or volunteering friends or relatives of the cases as control group [2, 4-6]. These participants are probably healthier and will therefore differ considerably from the cases. But also commonly used strategies, such as using matched controls, obtained from general practitioner databases, will not prevent information bias [1]. Studies considering self-limiting diseases such as gastroenteritis, might select a "help seeking" and therefore biased population. As a consequence, people using a PPI will be more inclined to consult medical help in case of gastroenteritis compared to randomly selected controls, even if they are matched.

Residual confounding might also have influenced previous study results, because dietary pattern, which has been shown to be the most important risk factors for Campylobacter and Salmonella infections has not been included in these studies [29, 30]. An association with dietary data 
Table 3 Association of use of proton pump inhibitors with bacterial gastrointestinal infections

$\begin{array}{lllll}\text { Number of cases } & \text { Number in cohort } & \text { Use of PPI }(\%) & \text { Infections } N=125 \text { OR }(95 \% \mathrm{CI}) & P \text { value }\end{array}$

Total cohort $N=12,515$

Current use of proton pump inhibitor

Univariate analysis $\quad 125$

Adjusted analysis-model $1 \quad 125$

12,515

$11.7^{*}$

$3.35(2.31 ; 4.87)$

$<0.001$

Adjusted analysis-model $2 \quad 125$

12,515

11.7 *

$4.03(2.77 ; 5.87)$

$<0.001$

Male only-model $2 \quad 53$

12,515

$11.7^{*}$

$6.14(3.81 ; 9.91)$

$<0.001$

Female only-model 2

5,113

$11.07(5.51 ; 22.24)$

$<0.001$

$72 \quad 7,402$

$12.7^{*}$

$3.83(1.96 ; 7.47)$

$<0.001$

Nested case-control analysis-all other participants of The Rotterdam Study as control group

Model 1: adjusted for sex, age

Model 2: adjusted for sex, age, cohort, calendar date, past use of proton pump inhibitors, current use of chronic medication, past use of H2receptor antagonists

* Percentage over 125 strata

$\dagger$ Percentage over 53 strata

* Percentage over 72 strata

was not observed in our study. Unfortunately, the number of missing dietary data in our study was rather high with as a consequence a large number of imputed data.

Observational studies may always suffer from bias and residual confounding. We had no data on other important risk factors for bacterial gastroenteritis, such as foreign travelling, eating in a restaurant, or contact with animals $[29,30]$.

We believe we used a comparable control group using participants with negative stool samples. However, results from a test-negative study design may also underestimate the risk, since a number of the controls with negative stool cultures may be false-negatives. They can be false-negative either because of low diagnostic sensitivity against the four bacterial species (e.g., too little material received, long transportation time, stool collected many days after onset of gastroenteritis) or because the patient suffered from other causes of bacterial gastroenteritis (diarrheagenic Escherichia coli or Clostridium difficile).

Furthermore, in elderly gastric acid secretion is impaired compared to younger aged individuals [31]. If gastric acid secretion is already impaired, PPIs will have a smaller effect. Therefore, the smaller risk estimate in our study might be explained by the fact that our population mainly consisted of elderly, in which gastric acid secretion is already impaired.

Because Salmonella species are less susceptible to $\mathrm{pH}$, the association between PPI therapy and gastroenteritis might be smaller for Salmonella species [19]. Unfortunately, however, the number of Salmonella infections in this study was too small to draw conclusions on this association.

During the study period the method of detection of enteric pathogens changed by the introduction of PCR, resulting in an increased sensitivity. To correct for an increased risk of false negative stool samples in the earlier years of the study, we included calendar date and cohort in the assessment of model design.

Although male gender has been shown to be an independent risk factor for bacterial gastroenteritis, it has not been shown before that the association between PPI therapy and bacterial gastroenteritis is much higher for males [32]. We were unable to explain this result by other covariables. Possibly there are unmeasured (hygiene related) behavioural aspects to explain this difference. In experimental studies a gender difference was observed between male and female neutrophils. Male neutrophils show higher responsiveness to stimulation with lipopolysaccharide and interferon- $\Upsilon$ [33]. Neutrophils play an important role in Salmonella and Campylobacter infections [22, 23]. The harmful effect of PPIs may therefore be greater for male than for female subjects. Of course, studies are needed to test this rather speculative hypothesis.

In conclusion, current PPI therapy was associated with an increased risk of bacterial gastroenteritis. We demonstrated that the effect is lower than previously assumed, by reducing the risk of information bias in our study design.

\section{Compliance with ethical standards}

Conflicts of interest All authors report no potential conflicts.

Open Access This article is distributed under the terms of the Creative Commons Attribution 4.0 International License (http://crea tivecommons.org/licenses/by/4.0/), which permits unrestricted use, distribution, and reproduction in any medium, provided you give appropriate credit to the original author(s) and the source, provide a link to the Creative Commons license, and indicate if changes were made. 


\section{References}

1. Neal KR, Scott HM, Slack RC, Logan RF. Omeprazole as a risk factor for campylobacter gastroenteritis: case-control study. BMJ. 1996;312:414-5.

2. Neal KR, Slack RC. Diabetes mellitus, anti-secretory drugs and other risk factors for campylobacter gastro-enteritis in adults: a case-control study. Epidemiol Infect. 1997;119:307-11.

3. Garcia Rodriguez LA, Ruigomez A, Panes J. Use of acid-suppressing drugs and the risk of bacterial gastroenteritis. Clin Gastroenterol Hepatol. 2007;5:1418-23.

4. Doorduyn Y, Van Pelt W, Siezen CL, Van Der Horst F, Van Duynhoven YT, Hoebee B, et al. Novel insight in the association between salmonellosis or campylobacteriosis and chronic illness, and the role of host genetics in susceptibility to these diseases. Epidemiol Infect. 2008;136:1225-34.

5. Doorduyn Y, Van Den Brandhof WE, Van Duynhoven YT, Breukink BJ, Wagenaar JA, Van Pelt W. Risk factors for indigenous Campylobacter jejuni and Campylobacter coli infections in The Netherlands: a case-control study. Epidemiol Infect. 2010;138:1391-404.

6. Doorduyn Y, Van Den Brandhof WE, Van Duynhoven YT, Wannet WJ, Van Pelt W. Risk factors for Salmonella Enteritidis and Typhimurium (DT104 and non-DT104) infections in The Netherlands: predominant roles for raw eggs in Enteritidis and sandboxes in Typhimurium infections. Epidemiol Infect. 2006;134:617-26.

7. Banatvala N, Cramp A, Jones IR, Feldman RA. Salmonellosis in North Thames (East), UK: associated risk factors. Epidemiol Infect. 1999;122:201-7.

8. Wu HH, Chen YT, Shih CJ, Lee YT, Kuo SC, Chen TL. Association between recent use of proton pump inhibitors and nontyphoid salmonellosis: a nested case-control study. Clin Infect Dis. 2014;59:1554-8.

9. Garcia Rodriguez LA, Ruigomez A. Gastric acid, acid-suppressing drugs, and bacterial gastroenteritis: how much of a risk? Epidemiology. 1997;8:571-4.

10. de Jongh E, Numans ME, de Wit NJ, Heemstra-Borst CG, Geijer RM, Burgers JS. [Summary of the Dutch College of General Practitioners' (NHG) practice guideline 'Gastric symptoms'] Samenvatting van de NHG-standaard 'Maagklachten'. Ned Tijdschr Geneeskd. 2013;157:A6101.

11. Shin JM, Sachs G. Pharmacology of proton pump inhibitors. Curr Gastroenterol Rep. 2008;10:528-34.

12. Huang JQ, Hunt RH. Pharmacological and pharmacodynamic essentials of $\mathrm{H}(2)$-receptor antagonists and proton pump inhibitors for the practising physician. Best Pract Res Clin Gastroenterol. 2001;15:355-70.

13. Laheij RJ, Sturkenboom MC, Hassing RJ, Dieleman J, Stricker $\mathrm{BH}$, Jansen JB. Risk of community-acquired pneumonia and use of gastric acid-suppressive drugs. JAMA. 2004;292:1955-60.

14. Tleyjeh IM, Bin Abdulhak AA, Riaz M, Alasmari FA, Garbati MA, AlGhamdi M, et al. Association between proton pump inhibitor therapy and clostridium difficile infection: a contemporary systematic review and meta-analysis. PLoS One. 2012;7:e50836.

15. Bavishi C, Dupont HL. Systematic review: the use of proton pump inhibitors and increased susceptibility to enteric infection. Aliment Pharmacol Ther. 2011;34:1269-81.
16. Bouwknegt M, van Pelt W, Kubbinga ME, Weda M, Havelaar AH. Potential association between the recent increase in campylobacteriosis incidence in the Netherlands and proton-pump inhibitor use an ecological study. Euro Surveill. 2014;19(32):21.

17. Leonard J, Marshall JK, Moayyedi P. Systematic review of the risk of enteric infection in patients taking acid suppression. Am J Gastroenterol. 2007;102:2047-56.

18. Sarker SA, Gyr K. Non-immunological defence mechanisms of the gut. Gut. 1992;33:987-93.

19. Foley SL, Johnson TJ, Ricke SC, Nayak R, Danzeisen J. Salmonella pathogenicity and host adaptation in chicken-associated serovars. Microbiol Mol Biol Rev. 2013;77:582-607.

20. Freedberg DE, Lebwohl B, Abrams JA. The impact of proton pump inhibitors on the human gastrointestinal microbiome. Clin Lab Med. 2014;34:771-85.

21. Masanta WO, Heimesaat MM, Bereswill S, Tareen AM, Lugert $\mathrm{R}$, Groß U, et al. Modification of intestinal microbiota and its consequences for innate immune response in the pathogenesis of campylobacteriosis. Clin Dev Immunol. 2013;2013:526860.

22. Kohler H, Sakaguchi T, Hurley BP, Kase BA, Reinecker HC, McCormick BA. Salmonella enterica serovar Typhimurium regulates intercellular junction proteins and facilitates transepithelial neutrophil and bacterial passage. Am J Physiol Gastrointest Liver Physiol. 2007;293:G178-87.

23. Kedika RR, Souza RF, Spechler SJ. Potential anti-inflammatory effects of proton pump inhibitors: a review and discussion of the clinical implications. Dig Dis Sci. 2009;54:2312-7.

24. Brophy S, Jones KH, Rahman MA, Zhou SM, John A, Atkinson $\mathrm{MD}$, et al. Incidence of Campylobacter and Salmonella infections following first prescription for PPI: a cohort study using routine data. Am J Gastroenterol. 2013;108:1094-100.

25. Hofman A, Brusselle GG, Darwish Murad S, van Duijn CM, Franco $\mathrm{OH}$, Goedegebure A, The Rotterdam Study, et al. objectives and design update. Eur J Epidemiol. 2016;2015(30):661-708.

26. Hubbard AE, Ahern J, Fleischer NL, Van der Laan M, Lippman SA, Jewell N, et al. To GEE or not to GEE: comparing population average and mixed models for estimating the associations between neighborhood risk factors and health. Epidemiology. 2010;21:467-74.

27. Gardiner JC, Luo Z, Roman LA. Fixed effects, random effects and GEE: what are the differences? Stat Med. 2009;28:221-39.

28. Stricker BH, Stijnen T. Analysis of individual drug use as a timevarying determinant of exposure in prospective population-based cohort studies. Eur J Epidemiol. 2010;25:245-51.

29. Domingues AR, Pires SM, Halasa T, Hald T. Source attribution of human campylobacteriosis using a meta-analysis of case-control studies of sporadic infections. Epidemiol Infect. 2012;140:970-81.

30. Domingues AR, Pires SM, Halasa T, Hald T. Source attribution of human salmonellosis using a meta-analysis of case-control studies of sporadic infections. Epidemiol Infect. 2012;140:959-69.

31. Hurwitz A, Brady DA, Schaal SE, Samloff IM, Dedon J, Ruhl CE. Gastric acidity in older adults. JAMA. 1997;278:659-62.

32. Skirrow MB. A demographic survey of campylobacter, salmonella and shigella infections in England A Public Health Laboratory Service Survey. Epidemiol Infect. 1987;99:647-57.

33. Aomatsu M, Kato T, Kasahara E, Kitagawa S. Gender difference in tumor necrosis factor-alpha production in human neutrophils stimulated by lipopolysaccharide and interferon-gamma. Biochem Biophys Res Commun. 2013;441:220-5. 